\title{
Hydrocarbon Binding Inside a Hexameric Pyrogallolarene Capsule
}

\author{
Liam Palmer, Julius Rebek, Jr.
}

\section{Supplementary Information}

\section{Experimental Section.}

${ }^{1} \mathrm{H}$ NMR spectra were recorded on a Brüker DRX-600 spectrometer operating at $600.13 \mathrm{MHz}$. Unless otherwise indicated, all spectra were obtained at $298 \mathrm{~K}$. 2D NOESY spectra were acquired with a delay time (d1) of $1.0 \mathrm{~s}$ and mixing times (d8) of 0.3-0.4 s. Proton spectra in non-deuterated solvents were acquired with a minimum of 128 scans and a sweep width of $18 \mathrm{ppm}$. A coaxial tube containing a deuterated solvent (e.g., benzene- $d_{6}$ ) was used to lock each sample. Proton integrals were determined using XWINNMR software. Since the high baseline intensity in each spectrum resulted from two or more solvent peaks, no solvent suppression was used.

Deuterated solvents were purchased from Cambridge Isotope Laboratories (Andover, MA). Chloroform- $d$ and benzene- $d_{6}$ samples were saturated with $\mathrm{H}_{2} \mathrm{O}$ and filtered before use. All other reagents (including all $n$-alkanes) were purchased from Aldrich (St. Louis, MO) and were used without further purification. methods. ${ }^{1}$

$\mathrm{C}_{11}$-footed pyrogallol[4]arene $\mathbf{1}$ were prepared using a modification of literature

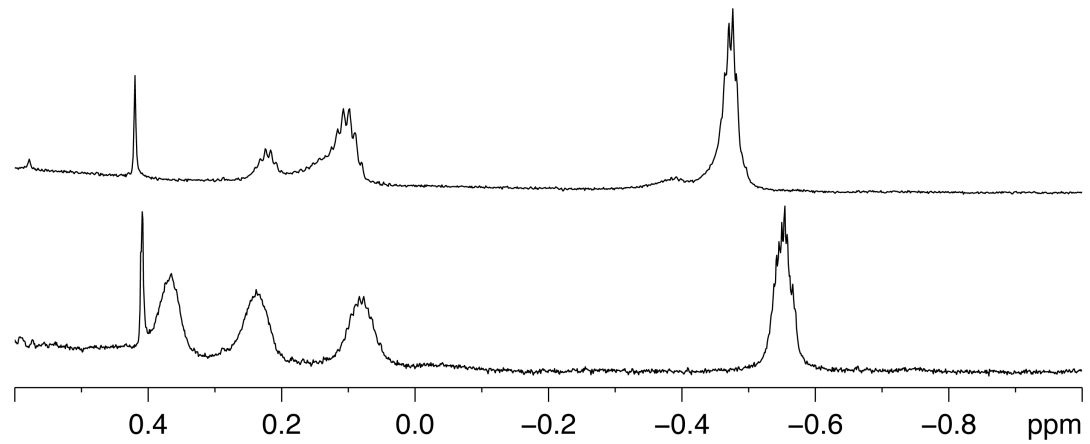

Figure S1. ${ }^{1} \mathrm{H}$ NMR of isooctane (2,2,4-trimethylpentane), top, and $n$-octane, bottom, with $\mathbf{1}_{6}$ at $298 \mathrm{~K}$. Only the upfield region is shown.

\footnotetext{
${ }^{1}$ Tunstad, L. M.; Tucker, J. A.; Dalcanale, E.; Weiser, J.; Bryant, J. A.; Sherman, J. C.; Helgeson, R. C.; Knobler, C. B.; Cram, D. J. J. Org. Chem. 1989, 54, 1305-1312.
} 
a

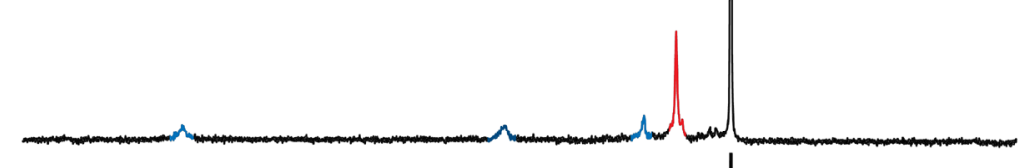

b

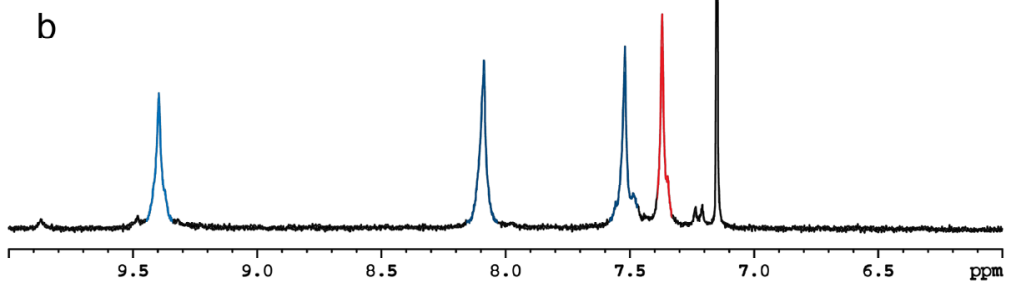

Figure S2. ${ }^{1} \mathrm{H}$ NMR of 1 in $n$-octane (a) after deuteration of exchangeable protons $\left(1-d_{12}\right)$ compared to (b) normal $\mathbf{1}$. The former sample was prepared by three times dissolving $\mathbf{1}$ in dichloromethane/ $\mathrm{CD}_{3} \mathrm{OD}$ and then evaporating to dryness. Only the downfield region is shown. The exchangeable $\mathrm{OH}$ protons are indicated in blue and the host aryl proton in red. The black peak is benzene used as an external lock solvent.

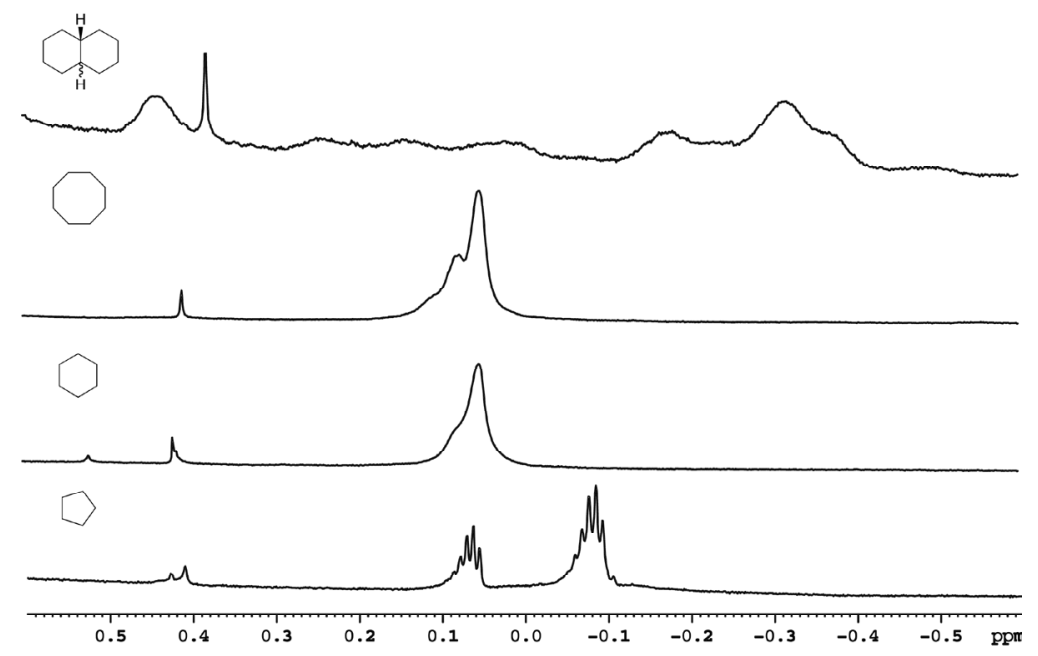

Figure S3. ${ }^{1} \mathrm{H}$ NMR of encapsulated cycloalkanes $(600 \mathrm{MHz}, 298 \mathrm{~K})$. From bottom to top: cyclopentane (may contain solvent impurity), cyclohexane, and cyclooctane, and decalin (mixture of cis and trans isomers). Only the upfield region is shown. 


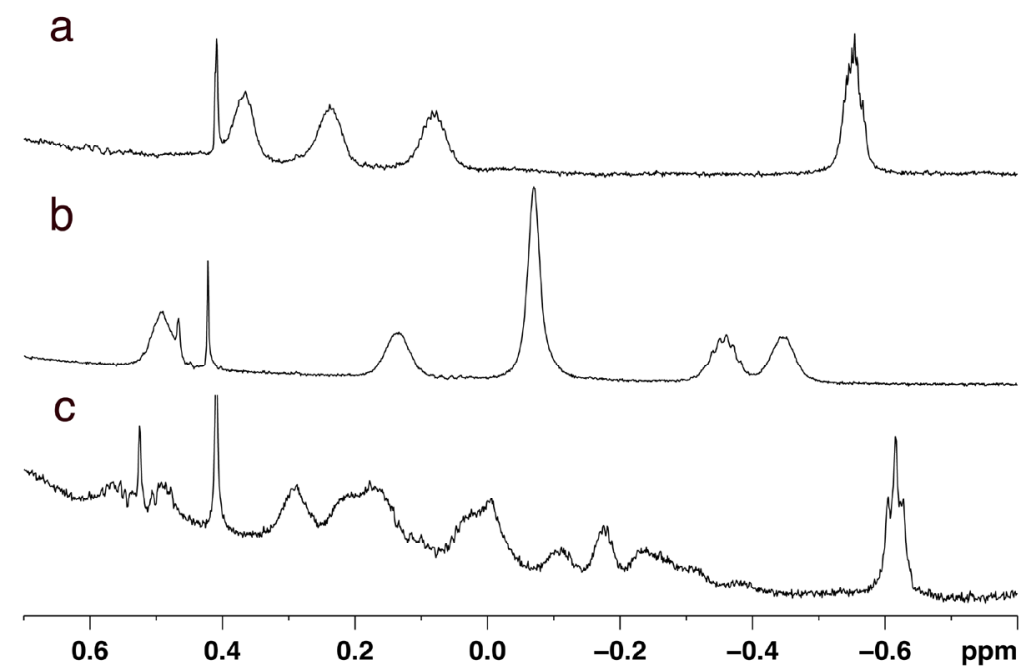

Figure S4. ${ }^{1} \mathrm{H}$ NMR of 1 in (a) pentane, (b) dodecane, and (c) 1:1 pentane/dodecane $(\mathrm{v} / \mathrm{v})$.

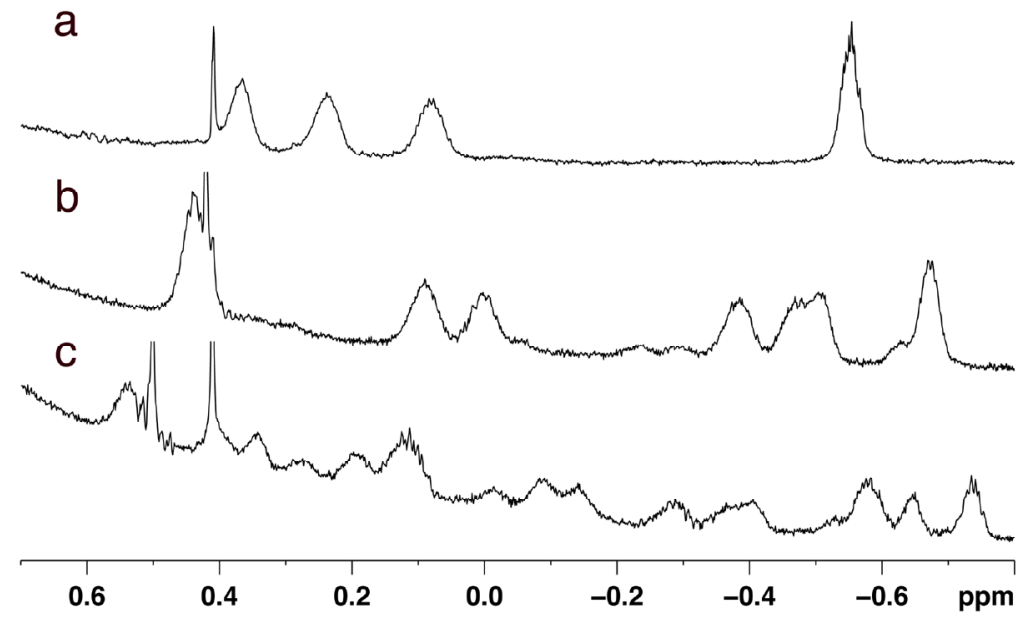

Figure S5. ${ }^{1} \mathrm{H}$ NMR of 1 in (a) pentane, (b) hexadecane, and (c) 1:1 pentane/hexadecane $(\mathrm{v} / \mathrm{v})$. 


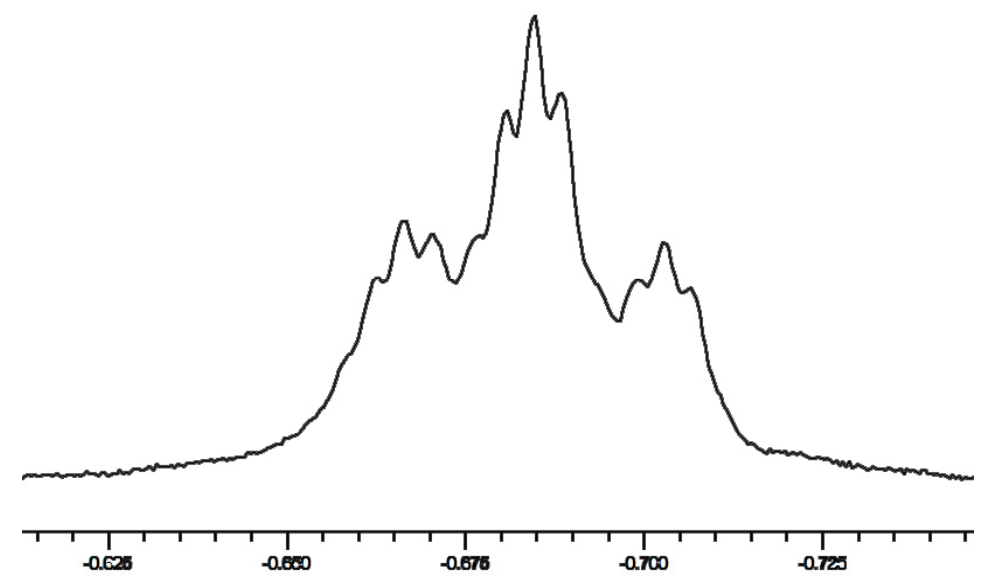

Figure S6. ${ }^{1} \mathrm{H}$ NMR of the terminal methyl resonance of $n$-octane encapsulated by $\mathbf{1}_{6}$ at 298 K. Only the upfield region is shown. The spectrum clearly shows a triplet of triplets with spin-spin coupling constants of 7.5 and $1.5 \mathrm{~Hz}$. 